University of Texas Rio Grande Valley

ScholarWorks @ UTRGV

Physics and Astronomy Faculty Publications and Presentations

$6-27-2017$

\title{
On orientation memory in high density polyethylene - Carbon nanofibers composites
}

\author{
Mircea Chipara \\ Brian Jones \\ Dorina M. Chipara \\ Jianhua Li \\ Karen Lozano
}

See next page for additional authors

Follow this and additional works at: https://scholarworks.utrgv.edu/pa_fac

Part of the Astrophysics and Astronomy Commons

\section{Recommended Citation}

Mircea Chipara, et. al., (2017) On orientation memory in high density polyethylene - Carbon nanofibers composites.E-Polymers17:4303. DOI: http://doi.org/10.1515/epoly-2016-0286

This Article is brought to you for free and open access by the College of Sciences at ScholarWorks @ UTRGV. It has been accepted for inclusion in Physics and Astronomy Faculty Publications and Presentations by an authorized administrator of ScholarWorks @ UTRGV. For more information, please contact justin.white@utrgv.edu, william.flores01@utrgv.edu. 


\section{Authors}

Mircea Chipara, Brian Jones, Dorina M. Chipara, Jianhua Li, Karen Lozano, Shah Valloppilly, and David Sellmyer 
Mircea Chipara*, Brian Jones, Dorina M. Chipara, Jianhua Li, Karen Lozano, Shah Valloppilly and David Sellmyer

\section{On orientation memory in high density polyethylene - carbon nanofibers composites}

DOI 10.1515/epoly-2016-0286

Received October 29, 2016; accepted January 4, 2017; previously published online February 1, 2017

Abstract: An orientation memory effect in high density polyethylene (HDPE) filled with vapor grown carbon nanofibers (VGCNF) is reported. Two-dimensional X-ray (2DXR) confirmed the reorientation of HDPE crystallites upon the uniaxial stretching of HDPE and HDPE filled by VGCNFs. This anisotropy of 2DXR spectra was decreased by heating all stretched samples (loaded or not loaded by VGCNFs) from room to the melting temperature of HDPE. Upon cooling these samples to room temperature, it was noticed that only the nanocomposite retained a weak partial (uniaxial) order, while HDPE showed a completely isotropic 2DXR spectrum. It was concluded that during the stretching of nanocomposites the crystallites and VGCNFs were aligned along the uniaxial stress. Upon heating, the crystalline phase was melted, while the orientation of the VGCNFs was not significantly disturbed. The recrystallization of the polymer started preferentially from the VGCNF - polymer interphase, resulting into an anisotropic crystalline structure.

Keywords: memory; polyethylene; two-dimensional X-ray spectra; vapor grown carbon nanofiber.

\footnotetext{
*Corresponding author: Mircea Chipara, The University of Texas Rio Grande Valley, Department of Physics, Edinburg, TX 78541, USA, e-mail:mircea.chipara@utrgv.edu; chipara@yahoo.com

Brian Jones: Bruker AXS Inc. 5465 East Cheryl Parkway, Madison, WI 53711-5373, USA

Dorina M. Chipara: The University of Texas Rio Grande Valley, Department of Physics, Edinburg, TX 78541, USA

Jianhua Li: Rice University, 6100 Main Street, Houston, TX 77005 , USA

Karen Lozano: The University of Texas Rio Grande Valley, Department of Mechanical Engineering, Edinburg, TX 78541, USA Shah Valloppilly and David Sellmyer: University of Nebraska, Department of Physics and Astronomy, Nebraska Center for Materials and Nanoscience, Lincoln, NE 68588, USA
}

\section{Introduction}

Polyethylene is a polymer with excellent structural properties. The loading of polyethylene with carbon nanostructures [single walled carbon nanotubes, multi wall carbon nanotubes, vapor grown carbon nanofibers (VGCNF), graphene, and fullerenes] affects the mechanical properties of the polymeric matrix, resulting typically in enhanced Young modulus, toughness, strain at fracture, ultimate stress and better thermal stability (1). Surface modifications of the nanofiler are also affecting the final features of the nanocomposite $(2,3)$. New features such as electrical conductivity (4) or enhanced thermal conductivity (5) may also be added by loading the polyethylene matrix with carbon nanostructures. Loading high density polyethylene (HDPE) with multiwall carbon nanotubes up to about $0.45 \%$ volume fraction resulted (6) in the increase of the Young modulus by about $20 \%$, of the strain at fracture by about $25 \%$ and of the toughness by about $30 \%$. Other authors reported (2), a minimum of the tensile strength in low density polyethylene at a loading of about $0.1 \%$ wt. multi wall carbon nanotubes and in linear low density polyethylene loaded with carbon nanotubes (7) and stiffness enhancements in HDPE loaded by multi wall carbon nanotube (8). Typically the addition of one dimensional carbon nanostructures decreases the elongation at break in polyethylene (PE) (2), result assigned to the poor dispersion of the one dimensional carbon nanostructure within PE or to nan incomplete wetting of the nanofiller by the polymeric matrix (9), which favors the delamination. Carbon nanofillers are also affecting the nucleation and crystallization processes in polymers (10). Uniaxial stresses align the macromolecular chains along the stretching direction (11, 12). In PE loaded with carbon nanofibers (CNF), the uniaxial extension propagates further by aligning also the one dimensional nanofiller along the stretching direction (13). Polymer processing may also contribute to the modifications of the physical and chemical features of polymers loaded with nanofillers (14).

The uniaxial stretching of polyethylene produces an anisotropic orientation of polymer crystallites $(15,16)$ and of nanofillers (13). 


\section{Experimental methods}

Chevron Phillips Chemical Company provided the highdensity polyethylene (HDPE) pellets (grade HXM-50100), characterized by a density of $948 \mathrm{~kg} / \mathrm{m}^{3}$. The polymer has been loaded with VGCNF type PR-24AG, from Pyrograf Products. HDPE-VGCNF nanocomposites have been obtained by melt mixing using a Haake Rheomix with twin counter-rotating screws. The melt mixing was done in air, at $150^{\circ} \mathrm{C}$ and 60 rotations per minute, for $10 \mathrm{~min}$. Hot pressed HDPE and HDPE-VGCNF nanocomposites were obtained by pressing the polymer at $150^{\circ} \mathrm{C}$ for $1 \mathrm{~min}$ at $100 \mathrm{kN}$ over an area of about $100 \times 100 \mathrm{~mm}$.

The samples were labeled as follows: HDPE for the pristine polymer, HDPE-VGCNF_xA for the sample containing $\mathrm{x} \%$ wt. VGCNF and not subjected to any mechanical solicitation, HDPE-VGCNF_xB for the as obtained samples subjected to a 500\% strain, and HDPE-VGCNF_xC for the as prepared sample subjected to a $1000 \%$ strain. In this study $\mathrm{x}=0 \%, 10 \%$ and $20 \%$ wt., respectively.

Wide angle X-ray scattering (WAXS) measurements have been performed using a Rigaku spectrometer (Rigaku Americas, TX, USA) and 2DXR diffraction spectra have been obtained by using a Bruker Discover 8 spectrometer (Bruker AXS, USA) equipped with the general area detector diffraction system (GADDS). Scanning Electron Microscopy photographs were obtained by using a Zeiss Merlin scanning electron microscope (SEM).

\section{Experimental results and discussions}

SEM photos of the as obtained nanocomposites HDPEVGCNF_20A (Figure 1A), and of stretched nanocomposites HDPE-VGCNF_20B (Figure 1B) and HDPE-VGCNF_20C (Figure 1C) confirmed that the VGCNFs are well dispersed within the HDPE matrix. The orientation of the VGCNFs along the external uniaxial stress is noticed. Additionally, the fragmentation of VGCNFs due to the applied uniaxial mechanical stress has been noticed.

The stress-strain dependence reported (17) in these samples reveals an unusual behavior as both the tensile strength and the elongation at break are increased due to the loading with nanofiller (17) (see Figure 2). Such unique behavior justifies a detailed analysis of the morphological modifications. SEM microscopy data suggests that the fragmentation of the VGCNFs allowed the slippage of the macromolecular chains, increasing the elongation at break. It is speculated that the mechanical processing generated defects on VGCNFs that decreased the stress required to break them.

WAXS measurements done with the Rigaku equipment revealed the typical lines due to crystalline HDPE (see Figure 3). The WAXS spectrum of pressed and aligned (up to a strain of 500\%) pristine HDPE is dominated by the lines corresponding to [110] and [220] reflections,

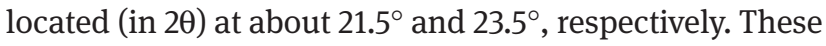
lines are consistent with the orthorhombic crystal cell of HDPE, as reported elsewhere (18). A (broad) hallo has been noticed in all samples at about $2 \theta_{\mathrm{a}} \times 20^{\circ}$, corresponding to an average intermolecular distance $d_{a} \approx 0.66 \lambda /$ $\sin \theta_{\mathrm{a}} \approx 0.0 .6 \mathrm{~nm}(19)$. This line is typically assigned to the amorphous phase in PE. However, the appearance of the [-201] peak upon mechanical stretching reported in (18), was not observed.

The upper part of Figure 4 includes the X-ray spectra of pristine HDPE and HDPE-VGFNF_20A. The sample filled with 20\% wt. VGCNFs exhibits an additional very weak and relatively broad line, located at $2 \theta=26^{\circ}$. This peak was noticed in HDPE-VGFNF_20A and assigned to VGCNFs. As shown in upper part of Figure 4, this line is not observed in the pristine polymer (see the red spectrum in top section of Figure 4). The inset of the top section of Figure 4 shows a magnified region of the WAXS spectrum that contains the line due to VGCNFs. The positions of the WAXS lines due to the polymeric matrix are the same for

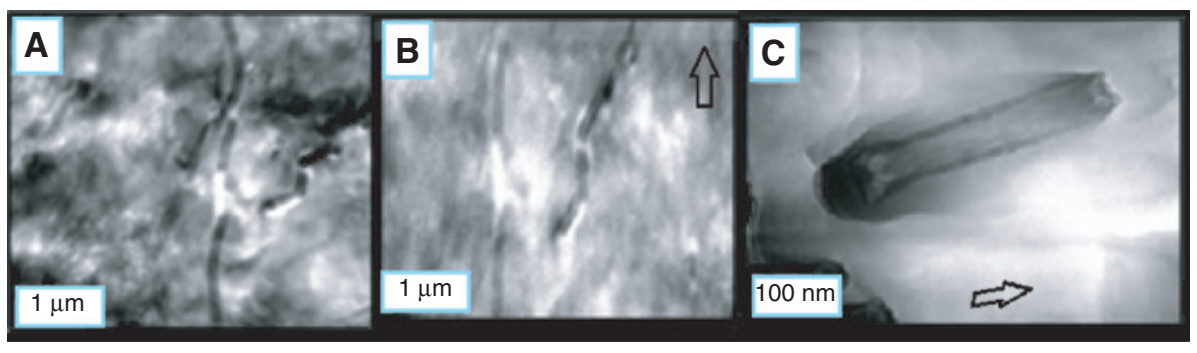

Figure 1: (A) SEM micrograph HDPE-VGCNF_20A (before stretching). (B) SEM of HDPE-VGCNF_20B (after a strain of 500\%). (C) SEM of HDPEVGCNF_20C (after a strain of 1000\%). The arrow indicates the stretching direction. 


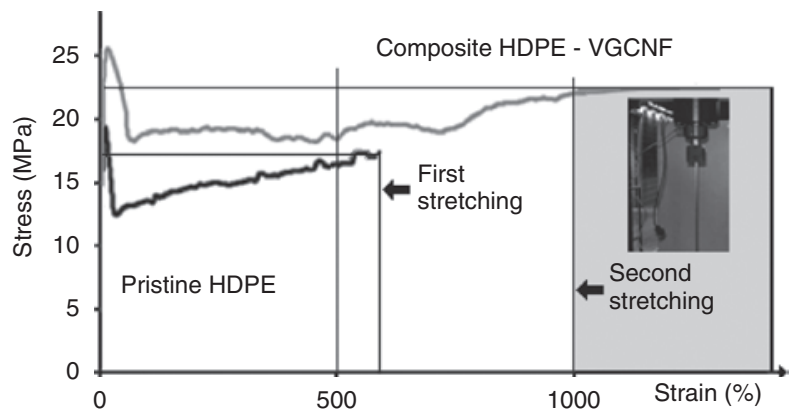

Figure 2: Stress-strain dependence for pristine HDPE and HDPE loaded by $20 \%$ wt. VGCNF.

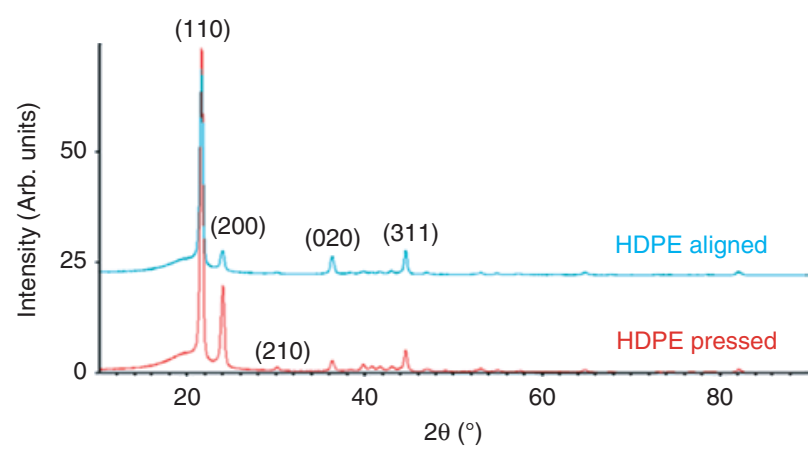

Figure 3: WAXS spectra of stretched (aligned) and pressed HDPE.

both spectra but small changes in the intensities of the components may be observed and assigned to small distortions of the crystalline cell of HDPE upon stretching. Nevertheless, it is important to notice that the reported strong decrease of the intensity of the [110] line was rather moderate for the samples investigated in this report (18, 20). The broad shoulder noticed at small angles is a fingerprint of the amorphous phase in HDPE. The lower component of Figure 4 shows the WAXS spectra of HDPE stretched (up to a strain of 500\%) recorded in two orientations. It is noticed that the relative positioning (between scanning direction and stretching direction) affects the line intensities of the polymeric matrices without producing significant shifts in the line position. The sample's orientation affects also the carbon nanotube peak, as noticed in the lower part of Figure 4. These observations indicate that both the macromolecular chains and the VGCNFs are statistically aligned along the stretching direction.

The large area detector of the Bruker Discover eight provides additional details about the anisotropy of the stretched samples. Figure 5 shows the large area detector WAXS spectrum of pristine HDPE for different values of the angle $\Phi$. The right side represents the integration upon $\Phi$ angles, which converts the left (2D) spectrum into a $\theta-2 \theta$
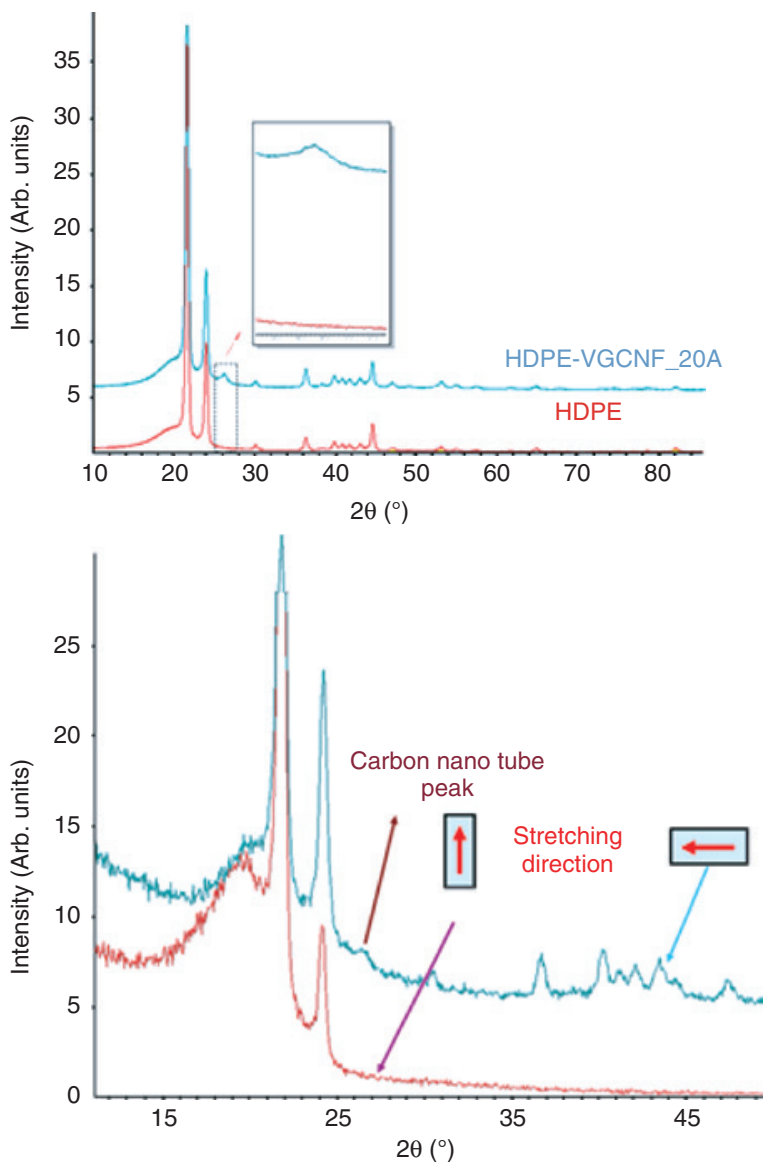

Figure 4: (Top) WAXS spectra of HDPE and HDPE-VGCNF_20A. (Lower) WAXS spectra of HDPE-VGCNF_20B in two configurations, parallel and perpendicular to the stretching direction.

spectrum, similar to the spectra shown in Figures 2-4. All reflections located on a circle of a given radius (see Figure 5) correspond to an unique angle $\theta$, and hence to a set of hkl numbers. From Figure 5 it is noticed that for pristine HDPE each circle has almost the same intensity (and width), indicating that the pristine HDPE is isotropic, i.e. the crystallites are randomly oriented in all directions.

The integration of the 2DXR spectra over different orientations $\Phi$, resulted in a collection of spectra, shown in the right panel of Figure 6 (for each angle $\Phi$ for the pristine and not stretched HDPE filled with 10\% VGCNF). It is noticed that all lines have the same position, amplitude, and width, irrespective on the $\varphi$ angle value, supporting the conclusion that the pristine HDPE sample is isotropic. Figure 6 collects the integrated 2DXR spectra, for various values of the angle $\Phi$, for pristine HDPE.

Figure 7 collects the integrated two-dimensional X-ray spectra, for various values of the angle $\Phi$, for HDPE filled by $10 \%$ VGCNF and not subjected to mechanical stresses. It is noticed that the spectra are isotropic and the presence 


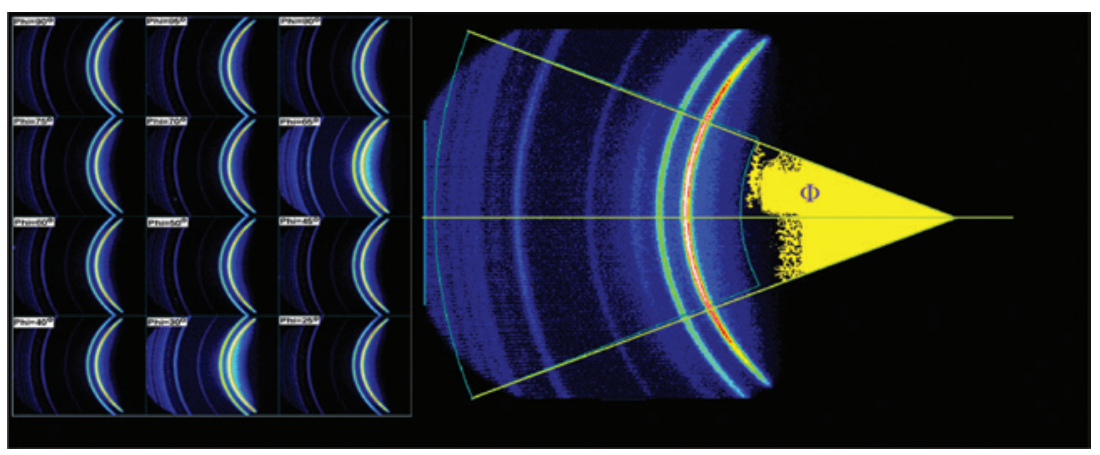

Figure 5: (Left side) GADDS spectra of pristine HDPE corresponding to various orientations. (Right side) The integration used to convert the two-dimensional X-ray spectrum into $2 \theta$ standard WAXS spectrum.

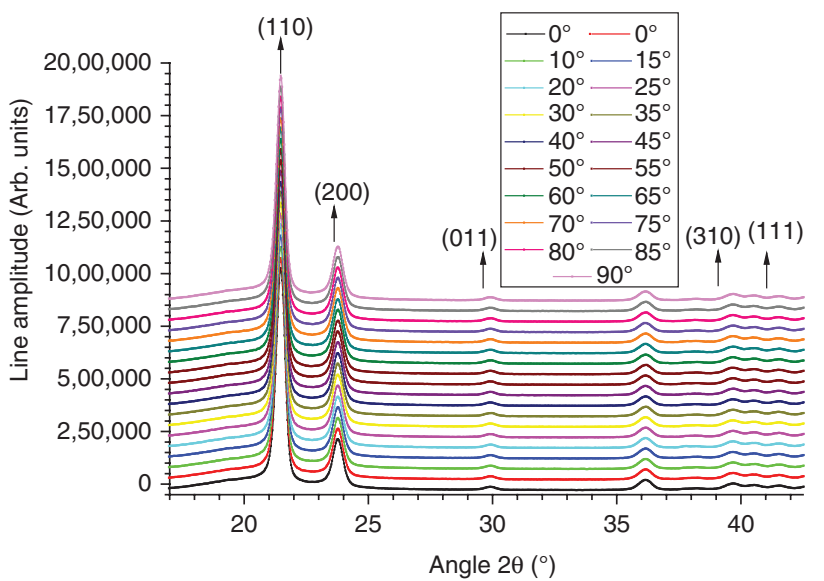

Figure 6: One-dimensional wide angle $X$-ray scattering on pristine HDPE not subjected to mechanical stress.

of VGCNF is represented by a very weak and broad line. The right side of Figure 7 shows the corresponding $\theta-2 \theta$ representation for various $\Phi$ angles. These dependencies show no anisotropy, indicating that the loading with VGCNFs did not affected the isotropic distribution of HDPE crystallites.
Figure 8 collects the two-dimensional X-ray spectra of pristine HDPE, after being subjected to a $500 \%$ strain, for various $\Phi$ values, at three temperatures (room temperature $77^{\circ} \mathrm{C}$ and $122^{\circ} \mathrm{C}$ ). It is observed that the intensity and width of each circle is no more constant (for a given constant radius), at any temperature. This indicates that the sample became anisotropic and explains why the $\theta-2 \theta$ spectrum may miss some reflections or may not show that the sample became anisotropic.

The lines assigned to the polymeric matrix (HDPE) are not shifted by the addition of VGCNF and their intensities are not significantly affected. A weak and broad line due to VGCNF is noticed around $2 \theta=26^{\circ}$. In conclusion, HDPE crystallites are not distorted by the presence of the nanofiller, suggesting that the nanofiller is avoiding the HDPE crystallites.

The effect of stretching on the two-dimensional X-ray spectrum of pristine HDPE is shown in the left panel Figure 8. By comparing these spectra, corresponding to various $\Phi$ angles with the corresponding spectra of pristine and not stretched HDPE shown in Figure 7 (left side) it is concluded that the $500 \%$ strain induced a clear
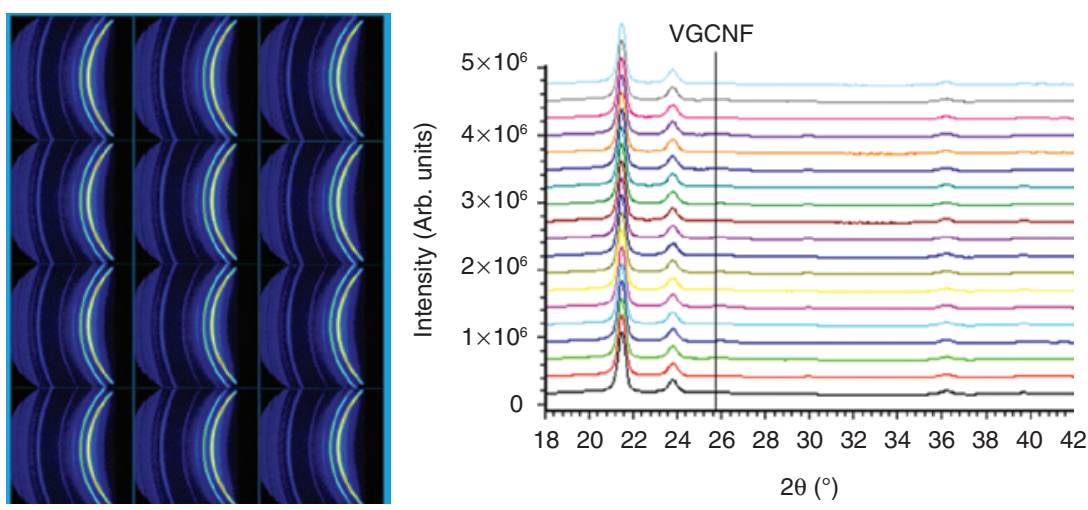

Figure 7: Two-dimensional X-ray spectra on HDPE loaded by $10 \%$ wt. VGCNF at room temperature (left) and the corresponding $2 \theta$ spectra for different orientations. 

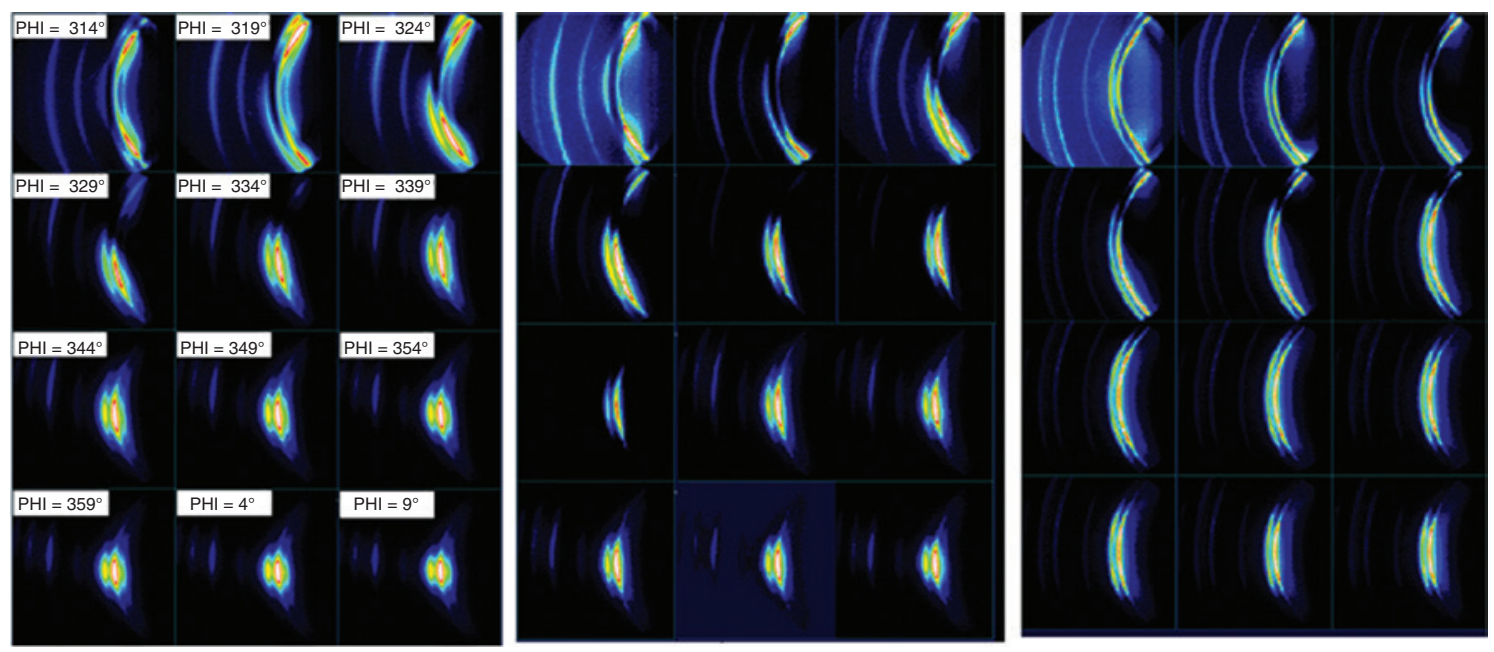

Figure 8: Two-dimensional X-ray spectra on pristine HDPE, subjected to mechanical stress at $500 \%$ strain and different temperatures. Left: room temperature. Middle: sample measures at $77^{\circ} \mathrm{C}$. Right. Sample measured at $122^{\circ} \mathrm{C}$.

anisotropy. The sample stretched at room temperature was then heated at $77^{\circ} \mathrm{C}$ and $122^{\circ} \mathrm{C}$. The effect of heating is also noticed from Figure 8. As the temperature at which the two-dimensional spectrum is recorded is increased, the anisotropy of the spectrum is decreased.

The anisotropy of pristine HDPE due to uniaxial stretching is supported by the data shown in Figure 9, where the dependence of the line position (top left side), line width (lower left side), line intensity (top right side), and line area (lower right side) on the angle $\Phi$ is represented for the most intense reflection [100] of pristine HDPE, both not stretched (squares) and strained up to $500 \%$ (circles). All spectra are recorded at room temperature.

As seen in Figure 10 (right side), the spectrum of the HDPE filled with 20\% VGCNF cooled down to room temperature (after stretching at 500\% strain and heating above $122^{\circ} \mathrm{C}$ ) is not isotropic.

It is noticed that the loading with VGCNF reduces the degree of the orientation of macromolecular chains along the stretching direction. This can explain the improved mechanical features of the nanocomposite and confirms the interactions between macromolecular chains and VGCNFs. Similar results have been obtained for HDPE filled with $10 \%$ VGCNF.

The main steps of the observed memory effect are: the pristine polymeric matrix is crystalline containing randomly oriented crystallites. The melt mixing of the polymeric with the nanofiller does not induce any anisotropy. The spectrum of the as obtained nanocomposites indicates randomly oriented crystallites. Eventually the nanofiller is also randomly dispersed within the polymer.
Upon stretching at room temperature of both pristine polymer and nanocomposites an anisotropic spectrum is noticed and assigned to the preferential orientation of polymer crystallites along the stretching direction. It is speculated that the distribution of orientation for nanofiller is also modified, i.e. the nanofiller (in our case VGCNFs) presents a certain preferential alignment along the stretching direction.

Heating the stretched samples (pristine polymer and nanocomposite) it is observed that the anisotropy decreases gradually and disappears close to the melting temperature of the polymeric matrix. It is speculated that while the crystallites are melted, the nanofiller preserves essentially its spatial distribution (uniaxial anisotropy).

Upon cooling from the melting temperature towards the room temperature the pristine polymer shows an isotropic spectrum at room temperature. However, the nanocomposite shows an anisotropic spectrum, reminiscent to the symmetry imposed by the external stress. This behavior is explained by the preferential grow of polymer crystallites near the nanofiler as well as by an orientation of these crystallites along the nanofiller. This feature is responsible for the observed orientation of polymer crystallites in cooled nanocomposites. Some crystallites that eventually are growing at sufficiently are distance from the nanofiller are expected to show no anisotropy. This proposed scheme is shown in Figure 11.

The heating above the melting temperature washes out the crystalline anisotropy but is not sufficient to erase the memory of VGCNF orientation. Consequently, after heating at $127^{\circ} \mathrm{C}$ and cooling slowly at room temperature the pristine HDPE is isotropic while the HDPE filled with $20 \%$ VGCNF retains a partial anisotropy. 

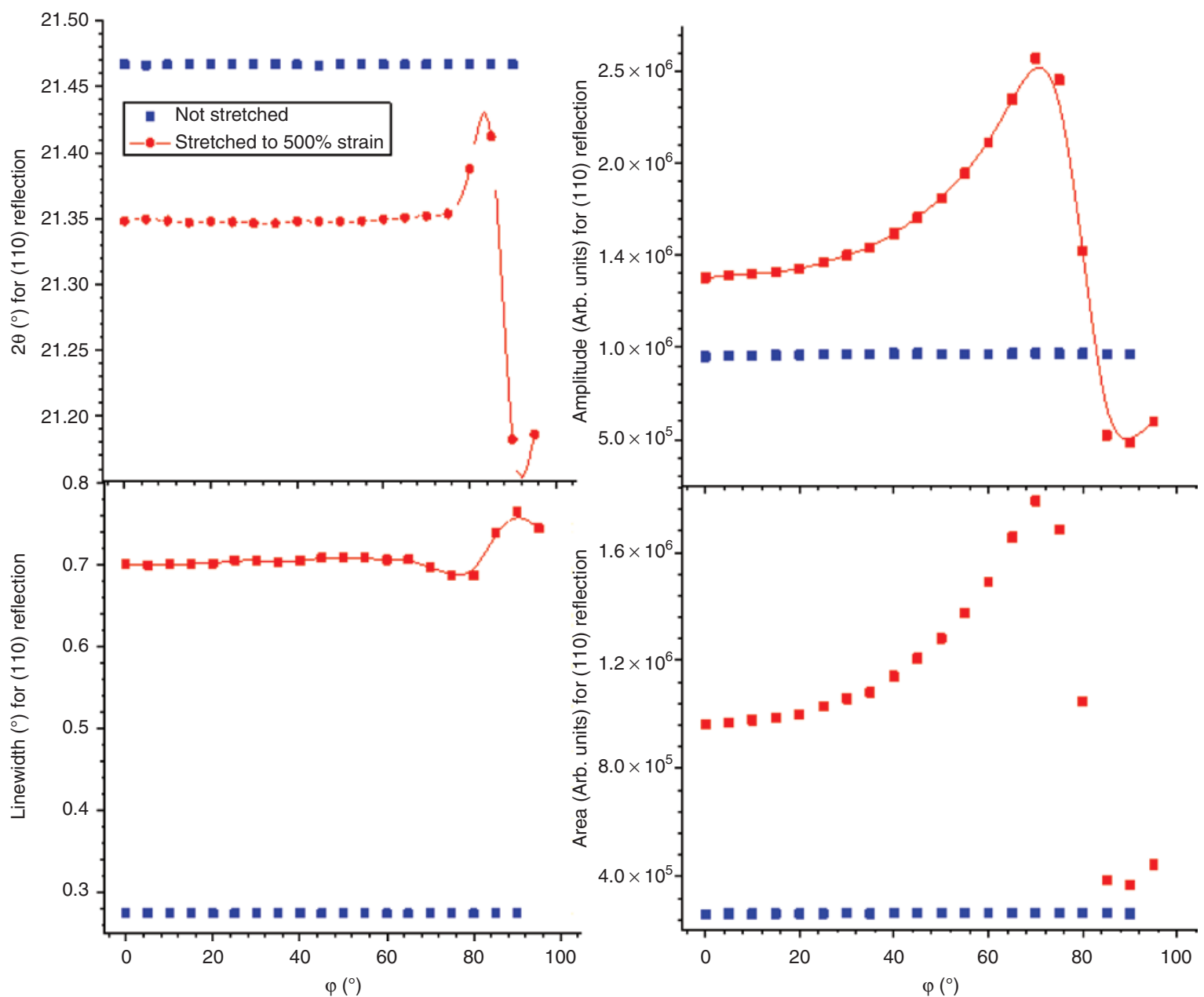

Figure 9: The dependence of the line parameters for the [100] reflection for pristine HDPE, at room temperature, not stretched (squares) and subjected to a $500 \%$ strain (circles) on the angle $\varphi$.
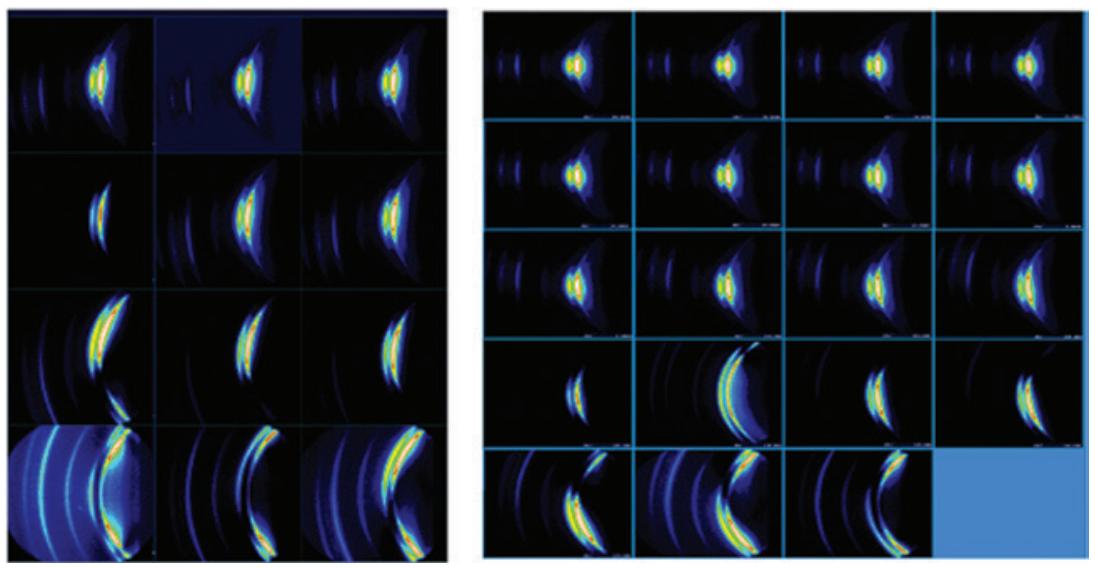

Figure 10: (Left) Two-dimensional X-ray diffraction on HDPE filled with $20 \%$ VGCNF, subjected to mechanical stress at $500 \%$ strain at room temperature. (Right) Two-dimensional X-ray diffraction on HDPE filled with $20 \%$ VGCNF, subjected to mechanical stress at $500 \%$ strain after heating at $122^{\circ} \mathrm{C}$ for $10 \mathrm{~min}$ and cooling to room temperature (recorded at room temperature). 


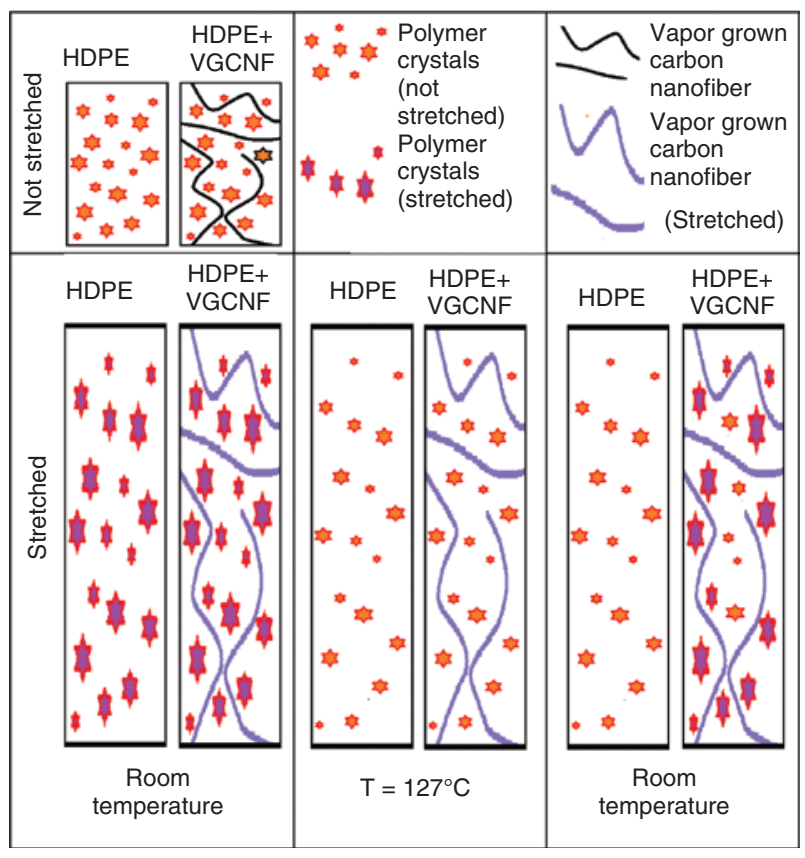

Figure 11: The main steps of the observed memory effect.

\section{Conclusions}

SEM micrographs confirmed the orientation of the VGCNFs along the direction of the uniaxial stress in HDPE-VGCNFs. Additionally, the micrographs suggested a fragmentation of VGCNFs during the mechanical testing which may explain the increase of the elongation at break in the samples filled with VGCNFs. It is suggested the melt mixing of HDPE and VGCNFs generated defects on the VGCNFs, which were responsible for the easy fracture of VGCNFs.

X-ray investigations on HDPE and HDPE filled with VGCNFs, subjected to uniaxial stretching are reported. It is concluded that pristine HDPE and HDPE loaded with VGCNF are isotropic. The uniaxial stretching is inducing an anisotropy, reflected by the two-dimensional X-ray spectra while almost absent in standard WAXS where the intensity of the scattered X-rays versus $2 \theta$ is recorded.

The uniaxial mechanical stretching below the melting temperature oriented both macromolecular chains and the nanofiller. Upon heating above the melting temperature, the crystalline phase was melted and the 2D X-ray spectrum (of HDPE) became isotropic for both pristine and HDPE-VGCNF samples. Cooling the stretched and melted HDPE frozen the crystallites in a random alignment. Nevertheless, the HDPE sample loaded with VGCNFs show again an anisotropy upon cooling below the melting temperature. This is explained by the fact that the CNF preserved their orientation during the melting and freezing steps and by the preferential growth of crystallites at the interface nanofiller-polymer.

This "structural" memory effect is thermally activated and in contrast with other memory effects does not involve a chemical bond. The memory effect provides a self-healing of the mechanical properties upon heating the nanocomposite slightly above the melting temperature. Applications are expected in components that require excellent mechanical properties. A simple application will be a differential strain sensor (in the area of civil engineering) where two identical dog bone shaped films one of pristine HDPE and other of HDPE filled with VGCNF can be used to sense the movement between two blocks/pieces. As the memory effect preserves a higher Young modulus for the sample loaded by VGCNFs, this sample will be stretched while the other will hang freely (after a cold stretching followed by melting). The advantage of this approach is that the sensor still operates even if the sensor is heated above the melting temperature (as in the case of direct exposure to sun during the day), if the sensing is done at a temperature lower than the melting temperature of the polymeric matrix. Theoretically, the sensor will work for several cooling/heating cycles. Some other applications may aim at structural materials for biological applications, where eventually a polymer with a lower melting temperature may be eventually used.

Acknowledgments: This research was supported at the University of Texas Rio Grande Valley by NSF PREM award DMR-1523577: UTRGV-UMN Partnership for Fostering Innovation by Bridging Excellence in Research and Student Success. The research was performed in part in the Nebraska Nanoscale Facility: National Nanotechnology Coordinated Infrastructure, the Nebraska Center for Materials and Nanoscience and Directorate for Mathematical and Physical Sciences, which are supported by the National Science Foundation under Award ECCS: 1542182 (University of Nebrasla, Lincoln), and the Nebraska Research Initiative.

\section{References}

1. Bocchini S, Frache A, Camino G, Claes M. Polyethylene thermal oxidative stabilisation in carbon nanotubes based nanocomposites. Eur Polym J. 2007;43:3222-35.

2. Abbasi SH, Al-juhani AA, Ul-hamid A, Hussein IA. Effect of aspect ratio, surface modification and compatibilizer on the mechanical and thermal properties of Idpe-mwcnt nanocomposites. e-Polymers. 2011;11(1):722-38. 
3. Shi X, Jiang B, Wang J, Yang Y. Influence of wall number and surface functionalization of carbon nanotubes on their antioxidant behavior in high density polyethylene. Carbon. 2012;50(3):1005-13.

4. Min C, Shen X, Shi Z, Chen L, Xu Z. The electrical properties and conducting mechanisms of carbon nanotube/polymer nanocomposites: a review. Polym Plast Technol Eng. 2010;49(12):1172-81.

5. Chiguma WEJ Jr, Johnson E, Pachamuthu A, Santos D. Electrically and thermally conducting nanocomposites for electronic applications. Materials (Basel). 2010;i:1478-96.

6. Kanagaraj S, Varanda FR, Zhil'tsova TV, Oliveira MSA, Simões JAO. Mechanical properties of high density polyethylene/carbon nanotube composites. Compos Sci Technol. 2007;67(15-16);3071-7.

7. Mezghani K, Farooqui M, Furquan S, Atieh M. Influence of carbon nanotube (CNT) on the mechanical properties of LLDPE/CNT nanocomposite fibers. Mater Lett. 2011;65(23-24):3633-5.

8. Tang W, Santare MH, Advani SG. M elt processing and mechanical property characterization of multi-walled carbon nanotube/ high density polyethylene (MWNT/HDPE) composite films. Carbon. 2003;41:2779-85.

9. Geoghegan M, Krausch G. Wetting at polymer surfaces and interfaces. Prog Polym Sci. 2003;28:261-302.

10. Haggenmueller R, Fischer JE, Winey KI. Single wall carbon nanotube/polyethylene nanocomposites: nucleating and templating polyethylene crystallites. Macromolecules. 2006;39(8):2964-71.

11. Che J, Locker CR, Lee S, Rutledge GC, Hsiao BS, Tsou AH. Plastic deformation of semicrystalline polyethylene by X-ray scattering: comparison with atomistic simulations. Macromolecules. 2013;46(13):5279-89.
12. Patil N, Balzano L, Portale G, Rastogi S. Influence of shear in the crystallization of polyethylene in the presence of SWCNTs. Carbon. 2010;48(14):4116-28.

13. Chipara M, Lozano K, Chipara MD. On the assessment of the orientation of carbon nanofibers dispersed within polyethylene by electron spin resonance spectroscopy. Carbon. 2007;45(13):2698-701.

14. Adhikari AR, Chipara M, Lozano K. Processing effects on the thermo-physical properties of carbon nanotube polyethylene composite. Mater Sci Eng A. 2009;526(1-2):123-7.

15. Grubb D, Prasad K. High-modulus polyethylene fiber structure as shown by X-ray diffraction. Macromolecules 1992;25(000):4575-82.

16. Matsuo M, Sawatari C. Elastic modulus of polyethylene in the crystal chain direction as measured by X-ray diffraction. Macromolecules. 1986;19:2036-40.

17. Shuying Yang KL, Taha-Tijerina J, Serrato-Diaz V, Hernandez K. Dynamic mechanical and thermal analysis of aligned vapor grown carbon nanofiber reinforced polyethylene. Compos Part B Eng. 2007;38(2):228-35.

18. Butler M, Donald A, Bras W, Mant GR, Derbyshire GE, Ryan AJ. A real-time simultaneous small-and wide-angle $X$-ray scattering study of in-situ deformation of isotropic polyethylene. Macromolecules. 1995;28(19):6383-93.

19. Rabiej S. WAXS investigations of the amorphous phase structure in linear polyethylene and ethylene-1-octene homogeneous copolymers. Fibres Text East Eur. 2005;13(5):30-4.

20. Galeski A, Bartczak Z, Argon AS, Cohen RE. Morphological alterations during texture-producing plastic plane strain compression of high-density polyethylene. Macromolecules. 1992;25(21):5705-18 